\title{
Parthenolide inhibits human lung cancer cell growth by modulating the IGF-1R/PI3K/Akt signaling pathway
}

\author{
LONGHUA SUN $^{1 *}$, WENXIN YUAN ${ }^{2 *}$, GUILAN WEN $^{1}$, BENTONG YU $^{3}$, FEI XU $^{1}$, XIN GAN $^{1}$, \\ JIANJUN TANG ${ }^{1}$, QINGHUA ZENG ${ }^{1}$, LANLAN ZHU ${ }^{1}, \mathrm{CHUANHUI} \mathrm{CHEN}^{1}$ and WEI ZHANG ${ }^{1}$ \\ Departments of ${ }^{1}$ Pulmonary and Critical Care Medicine, ${ }^{2}$ Ultrasonography and ${ }^{3}$ Thoracic Surgery, \\ The First Affiliated Hospital of Nanchang University, Nanchang, Jiangxi 330006, P.R. China
}

Received December 5, 2019; Accepted May 15, 2020

DOI: $10.3892 /$ or.2020.7649

\begin{abstract}
Lung cancer is the leading cause of cancer-associated mortality worldwide. Parthenolide (PTL), a natural product extracted from the plant Tanacetum parthenium, (a flowering plant in the daisy family, Asteraceae) has been reported to inhibit cancer cell growth, including that of human lung cancer. However, the underlying mechanisms by which PTL exerts its anticancer effect have remained to be fully elucidated. In the present study, Cell Counting Kit-8 and colony formation assays were used to assess the effect of PTL to inhibit cell proliferation, a wound-healing assay was performed to assess cell migration and western blot analysis and PCR were employed to reveal the molecular mechanisms by which PTL inhibits human lung carcinoma cell growth. The results indicated that PTL substantially inhibited cell proliferation and migration in two lung cancer cell lines A549 and H1299. Mechanistically, the phosphorylation of insulin-like growth factor 1 receptor (IGF-1R), Akt and forkhead box $\mathrm{O} 3 \alpha$ (FoxO3 $\alpha$ ) was blocked by PTL. Furthermore, IGF-1-induced Akt [protein kinase B or (PKB)] and FoxO3 $\alpha$ phosphorylation were also inhibited by PTL treatment. In addition, PTL significantly suppressed lung cancer growth in a subcutaneous xenograft mouse model. Taken together, the present in vivo and in vitro results indicate that PTL may suppress lung cancer growth through inhibiting IGF-1R-mediated PI3K/Akt/FoxO3 $\alpha$ signaling, suggesting
\end{abstract}

Correspondence to: Dr Wei Zhang, Department of Pulmonary and Critical Care Medicine, The First Affiliated Hospital of Nanchang University, 17 Yongwai Zheng Street, Nanchang, Jiangxi 330006, P.R. China

E-mail: zhangweiliuxin@163.com

*Contributed equally

Abbreviations: PTL, parthenolide; NSCLC, non-small cell lung cancer; IGF-1, insulin-like growth factor 1; IGF-1R, insulin-like growth factor 1 receptor; PI3K, phosphoinositide 3-kinase

Key words: lung cancer, parthenolide, migration, proliferation, IGF-1R that PTL may be an attractive candidate for the treatment of lung cancer.

\section{Introduction}

Lung cancer is the leading cause of cancer-associated mortality in males and females worldwide (1). Non-small cell lung cancer (NSCLC) is the most common lung cancer type, accounting for $\sim 85 \%$ of all lung cancers $(2,3)$. However, NSCLC is relatively resistant to chemotherapy and radiotherapy and the prognosis is poor, with an estimated overall 5-year survival of $<15 \%$ (4). Therefore, it is a matter of great urgency to develop effective treatment strategies as well as discovering novel therapeutic agents to treat lung cancer.

Traditional Chinese medicines (TCMs) obtained from plants have been widely considered as attractive sources of anticancer drugs owing to fewer side effects as compared with certain other chemotherapeutics $(5,6)$. It has been estimated that nearly half of the modern medicines in clinical use are small molecules derived from natural products (7), many of which exhibit extensive activities by downregulating the signaling cascades of growth factors, such as insulin-like growth factor (IGF). Among them, parthenolide (PTL), which is derived from the plant feverfew (Tanacetum parthenium; a flowering plant in the daisy family, Asteraceae), has been reported to exhibit a variety of biological activities, including antioxidant, anti-inflammatory and antitumor effects against various cancer types $(8)$, including colorectal $(9,10)$, pancreatic (11) and breast cancer $(12,13)$. These effects may be exerted through suppressing the proliferation, migration and invasion, and inducing apoptosis of cancer cells. Of note, recent studies have indicated that PTL also exhibits anticancer activity in lung cancer $(14,15)$. However, the exact mechanisms of action have remained largely elusive.

Signaling through receptor tyrosine kinase pathways is largely involved in tumor growth and progression (16). Accumulating evidence suggests that IGF signaling, which is comprised of the IGF ligand and IGF receptor (IGF-R), contributes to cell proliferation, differentiation and progression of numerous tumor types (17). Numerous studies have indicated that IGF-1R is overexpressed in numerous cancer types, including breast, prostate and colon cancers (17), and upregulation of IGF-1R results in chemotherapy resistance $(17,18)$. On 
the other hand, high expression levels of IGF-1, a ligand of IGF-1R, are closely associated with increased risk of multiple types of cancer (19). IGF-1 activates IGF-1R and then phosphorylates the downstream substrates through tyrosine kinase activity, resulting in activation of various downstream signaling pathways, including mitogen-activated protein kinases (MAPKs) and phosphoinositide 3-kinase (PI3K)/protein kinase B (Akt) signaling cascades (20). Therefore, developing IGF-1R-targeted inhibitors to interfere with IGF-1R-induced downstream signaling cascades has long been recognized as a potentially effective treatment strategy for various cancer types.

Over-activation of PI3K/Akt signaling cascades leads to the activation or inactivation of numerous downstream cytoplasmic and nuclear substrates, including forkhead box $\mathrm{O} 3$ (FoxO $3 \alpha$ ), which belongs to a family of transcription factors (21). FoxO3 $\alpha$ is a tumor suppressor that inhibits cell growth and induces apoptosis by regulating gene transcription and protein synthesis associated with tumorigenesis and cancer progression $(22,23)$. Activation of PI3K/Akt signaling following growth factor stimulation causes phosphorylation of FoxO3 $\alpha$, which leads to the export from the nucleus to the cytoplasm, thereby inhibiting its transcriptional activity and contributing to cell growth (22).

The present study aimed to demonstrate the anticancer effects of PTL. By using in vitro and in vivo analyses, the effects of PTL on the proliferation and migration of human lung cancer cells were investigated and the underlying molecular mechanisms were explored.

\section{Materials and methods}

Materials. Phospho-IGF-1R antibody (cat. no. 3024), IGF-1R antibody (cat. no. 9750), phospho-Akt antibody (cat. no. 4060), Akt antibody (cat. no. 4685), phospho-FoxO3 $\alpha$ antibody (cat. no. 9465), FoxO3 $\alpha$ antibody (cat. no. 2497), MMP-2 antibody (cat. no. 87809), MMP-9 antibody (cat. no. 13667), proliferating cell nuclear antigen (PCNA) antibody (cat. no. 13110), $\beta$-actin antibody (cat. no. 4970) and goat anti-rabbit horseradish peroxidase (HRP)-linked IgG antibody (cat. no. 7074) were purchased from Cell Signaling Technology, Inc. Ki67 antibody (cat. no. 27309-1-AP) was purchased from Proteintech Group Inc. IGF-1, PTL and LY294002 (an inhibitor of PI3K) were purchased from Sigma Aldrich; Merck KGaA. RPMI-1640 medium and fetal bovine serum (FBS) were purchased from Gibco (Thermo Fisher Scientific, Inc.).

Cell culture. Human NSCLC cell lines A549 and H1299 cells were purchased from the Cell Bank of the Chinese Academy of Sciences. The cells were cultured in RPMI-1640 medium supplemented with $100 \mathrm{U} / \mathrm{ml}$ penicillin/streptomycin and $10 \%$ FBS and were maintained at $37^{\circ} \mathrm{C}$ in a humidified atmosphere containing $5 \% \mathrm{CO}_{2}$.

Cell proliferation assay. A Cell Counting Kit-8 (CCK-8) assay was used to assess the inhibitory effects of PTL. In brief, $3 \times 10^{3}$ cells/well were seeded into 96-well plates. After $24 \mathrm{~h}$, the cells were treated with PTL at various concentrations for the indicated durations as specified in the Results. Following incubation, CCK-8 reagents were added and the cells were incubated for $1 \mathrm{~h}$ at room temperature. Cell proliferation was determined by measuring the optical density at $450 \mathrm{~nm}$ using an ELISA plate reader (Olympus Corp.).

Wound healing assay. Cells were seeded in 6-well plates at a concentration of $2 \times 10^{5}$ cells per well. Once they reached $80-90 \%$ confluence, the cells were serum-starved for $24 \mathrm{~h}$ and a wound on the midline of each well was then created by scraping the cell monolayer using a sterile $200-\mu 1$ pipette tip. The cells were then washed with PBS to rinse away any detached cells. Images of the wound margins were captured. Subsequently, serum-free medium containing the indicated drugs was added to each well. After incubation for $48 \mathrm{~h}$, images of the same area of the wound margins as those prior to the application of PTL were captured. To analyze the relative wound closure, the ratio between the unhealed wound area at a given time point and the original wound area $\mathrm{x} 100 \%$ was calculated using ImageJ software version $1.47 \mathrm{t}$ (National Institutes of Health).

Colony formation assay. The colony formation assay was performed according to the protocol of a previous study (24). In brief, $2 \times 10^{2}$ cells were cultured in a 6 -well plate and were treated with $10 \mu \mathrm{M}$ PTL. The medium containing PTL was replenished every three days. After a 2-week treatment, the cells were fixed with $4 \%$ paraformaldehyde and then stained with $0.1 \%$ crystal violet for $10 \mathrm{~min}$ at room temperature. The number of colonies containing $>50$ cells were counted using Image J software version 1.47t (National Institutes of Health).

Reverse transcription-quantitative PCR (RT-qPCR). After treatment, the total RNA was isolated by using TRIzol ${ }^{\mathrm{TM}}$ reagent (Thermo Fisher Scientific, Inc.). Reverse transcription into cDNA was performed using the PrimeScript ${ }^{\mathrm{TM}}$ RT Reagent Kit with gDNA Eraser (Perfect Real Time) according to the manufacturer's instructions (Takara Biotechnology Co., Ltd.). qPCR analysis was performed using TB Green ${ }^{\mathrm{TM}}$ Premix Ex Taq ${ }^{\mathrm{TM}}$ II (Tli RNaseH Plus) (Takara Biotechnology Co., Ltd.) on the StepOnePlus ${ }^{\mathrm{TM}}$ Real-Time PCR System (Thermo Fisher Scientific, Inc.) to determine the expression of matrix metalloproteinase (MMP)-2, MMP-9 and GAPDH by using the following primers: MMP-2, 5'-CCGTCGCCCATC ATCAAGTTC-3' (sense) and 5'-GCAGCCATAGAAGGT GTTCAGG-3' (antisense); MMP-9, 5'-TGGTCCTGGTGC TCCTGGTG-3' (sense) and 5'-GCTGCCTGTCGGTGAGAT TGG-3' (antisense); GAPDH, 5'-ACAACTTTGGTATCGTGG AAGG-3' (sense) and 5'-GCCATCACGCCACAGTTTC-3' (antisense). The reaction volume consisted of $20 \mu 1$, containing $2 \mu 1$ template cDNA, $10 \mu 1$ TB Green ${ }^{\mathrm{TM}}$ Premix Ex Taq $^{\mathrm{TM}}$ II (Tli RNaseH Plus), $1 \mu \mathrm{l} 10 \mu \mathrm{M}$ each primer, and $6 \mu \mathrm{lddH_{2 }} \mathrm{O}$. The amplification program included 1 cycle of $95^{\circ} \mathrm{C}$ for $30 \mathrm{sec}$ (initial denaturation), followed by 40 cycles of $95^{\circ} \mathrm{C}$ for $5 \mathrm{sec}$ (denaturation) and $60^{\circ} \mathrm{C}$ for $30 \mathrm{sec}$ (DNA synthesis). The relative mRNA expression levels were analyzed using the $2^{-\Delta \Delta \mathrm{Cq}}$ method (25).

Immunofluorescence. Cells were cultured and treated with PTL in confocal chambers. Then cells were then fixed with $4 \%$ paraformaldehyde for $15 \mathrm{~min}$, followed by permeabilization 
with $0.25 \%$ Triton $\mathrm{X}-100$ for $15 \mathrm{~min}$ at room temperature. Cells were then blocked with $1 \%$ bovine serum albumin (Biosharp) and incubated with anti-Ki-67 antibody overnight at $4^{\circ} \mathrm{C}$. Alexa Fluor 568-conjugated secondary antibodies (dilution 1:500; Thermo Fisher Scientific, Inc.) were applied for $2 \mathrm{~h}$ at room temperature, followed by nuclear counterstaining with DAPI at room temperature. Images were captured using an inverted microscope (magnification, x200) with fluorescence filters (Olympus Corp.).

Western blot analysis. After treatment, cells were lysed with SDS lysis buffer (Beyotime Institute of Biotechnology) containing protease inhibitor cocktail (Roche Diagnostics). Total protein was extracted and the bicinchoninic acid protein assay (Beyotime Institute of Biotechnology) was performed to determine the protein concentration. Equal amounts of proteins $(20 \mu \mathrm{g})$ were separated by $10 \%$ SDS-PAGE and transferred to nitrocellulose membranes (EMD Millipore). After blocking with 5\% non-fat dry milk for $1 \mathrm{~h}$ at room temperature, the membranes were incubated with the following primary antibodies overnight at $4^{\circ} \mathrm{C}$ : PNCA (dilution 1:2,000), phospho-Akt (dilution 1:2,000), Akt (dilution 1:4,000), phospho-IGF-1R (dilution 1:2,000), IGF-1R (dilution 1:2,000), phospho-FoxO3 $\alpha$ (dilution 1:2,000), FoxO3 $\alpha$ (dilution 1:2,000), MMP-2 (dilution 1:2,000), MMP-9 (dilution 1:2,000) and $\beta$-actin (dilution 1:4,000). After antibody binding, the membranes were washed and incubated with HRP-linked secondary antibodies (dilution 1:10,000) for $2 \mathrm{~h}$ at room temperature. The labeled proteins were visualized using enhanced chemiluminescence reagent (Pierce; Thermo Fisher Scientific, Inc.) and signals were quantified by using ImageJ software version $1.47 \mathrm{t}$ (National Institutes of Health).

Mouse subcutaneous xenografts and treatments. Ten male $\mathrm{BALB} / \mathrm{c}$ nude mice (age, 5 -weeks; weight, $18 \pm 0.2 \mathrm{~g}$ ) were obtained from Hunan Silaikejingda Laboratory Animal. All animal procedures were in accordance with the protocols approved by the Ethics Committee of the First Affiliated Hospital of Nanchang University (Nanchang, China). A total of $5 \times 10^{6}$ in $100 \mu 1$ A549 cells were subcutaneously injected into the right flank of nude mice. After a 7-day growth (tumor size was $250-300 \mathrm{~mm}^{3}$ ), the mice were randomly assigned to 2 groups ( $\mathrm{n}=5$ per group). The control group of mice was treated with saline, while the experimental group was intraperitoneally injected with PTL $(10 \mathrm{mg} / \mathrm{kg}$ ) daily for 24 days according to a previous study (26). The mice were anesthetized with $2 \%$ sodium pentobarbital $(10 \mathrm{ml} / \mathrm{kg})$ by intraperitoneal injection, and then sacrificed by cervical dislocation to dissect and weigh the tumors. The body weight was determined and the tumor volume $(\mathrm{V})$ was calculated every 4 days based on the following formula: $\mathrm{V}=(\text { the shortest diameter })^{2} \mathrm{x}($ the longest diameter) $\mathrm{x} 0.5$.

Statistical analysis. Values are expressed as the mean \pm standard error of the mean from at least three independent experiments. Statistical analyses were performed using GraphPad Prism 6.0 (GraphPad Software, Inc.) and comparisons were made by one/two-way analysis of variance with Bonferroni's multiple-comparison tests or Students' t-test. $\mathrm{P}<0.05$ was considered to indicate statistical significance.

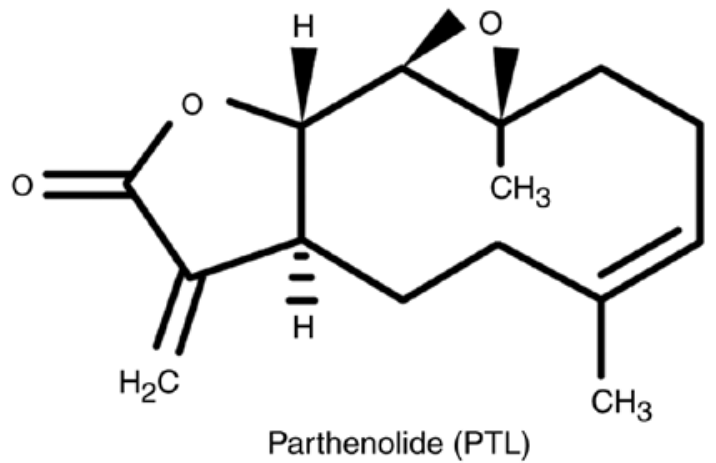

Figure 1. Chemical structure of parthenolide $\left(\mathrm{C}_{15} \mathrm{H}_{20} \mathrm{O}_{3}\right)$.

\section{Results}

PTL inhibits the growth of lung cancer cells. The chemical structure of PTL is presented in Fig. 1. To determine the effect of PLT on cancer cell proliferation, the human lung cancer cell lines A549 and H1299 were used in the present study. The cells were treated with increasing concentrations of PTL $(0,1,5,10$, $20,30$ and $50 \mu \mathrm{M})$ for $48 \mathrm{~h}$ and then assessed by the CCK-8 assay. PTL treatment decreased the cell viability of A549 and H1299 cells in a dose-dependent manner (Fig. 2A and B). Of note, at concentrations of $<5 \mu \mathrm{M}$, PTL had no noticeable effect, while significant inhibition was observed above the concentration of $5 \mu \mathrm{M}$ in both cell lines (Fig. 2A and B). Next, the impact of PTL treatment for different durations on A549 and H1299 cell viability was assessed. The cells were treated with $10 \mu \mathrm{M}$ PTL for $12,24,36$ or $48 \mathrm{~h}$ and the results indicated that PTL treatment for $24 \mathrm{~h}$ or above significantly inhibited the growth of A549 and H1299 cells in a time-dependent manner (Fig. 2C and D).

To further confirm the growth inhibition effects of PTL on lung cancer cells, a colony formation assay was performed. Consistent with the results of the CCK-8 assay, PTL markedly suppressed colony formation of A549 and H1299 cells and the number of colonies was significantly reduced (Fig. 2E and F). These results suggest that PTL exerts a significant growth inhibitory effect on lung cancer cells.

PTL inhibits the expression of proliferation markers in lung cancer cells. Nuclear antigen Ki67 has long been documented as one of the most wildly used markers of proliferation in oncology (27). Therefore, the PTL-mediated proliferation inhibition was further investigated by detecting the expression level of Ki67 in both lung cancer cell lines. An immunostaining assay was performed to evaluate the effect of PTL on cell proliferation (Fig. 3). PLT significantly inhibited the expression of Ki67 in A549 cells (Fig. 3A and C). Similar results were observed in H1299 cells (Fig. 3B and C). In addition, the expression of another cell proliferation marker, namely proliferating cell nuclear antigen (PCNA), was detected. PTL treatment for either 24 or $48 \mathrm{~h}$ significantly reduced PCNA expression in both cell lines (Fig. 3D-F). Taken together, these results suggest that PTL significantly inhibits the proliferation of lung cancer cells.

PTL suppresses the migration of lung cancer cells. Since migration is a crucial factor that contributes to cancer 

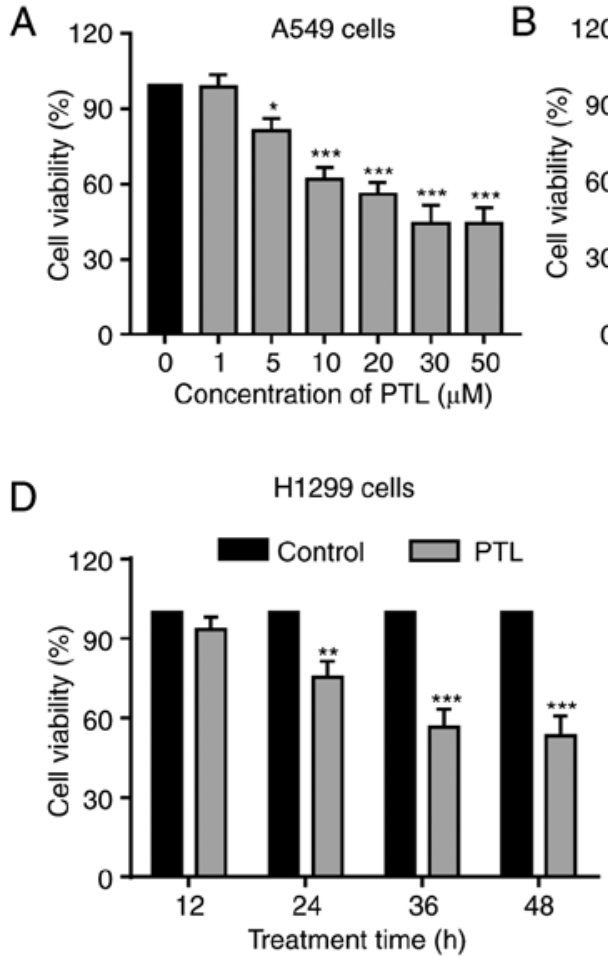

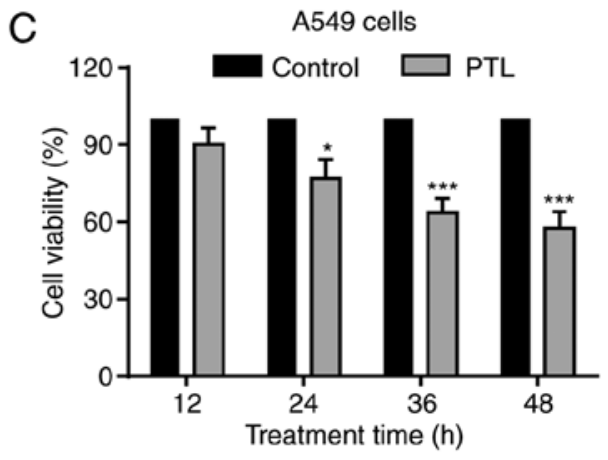

E
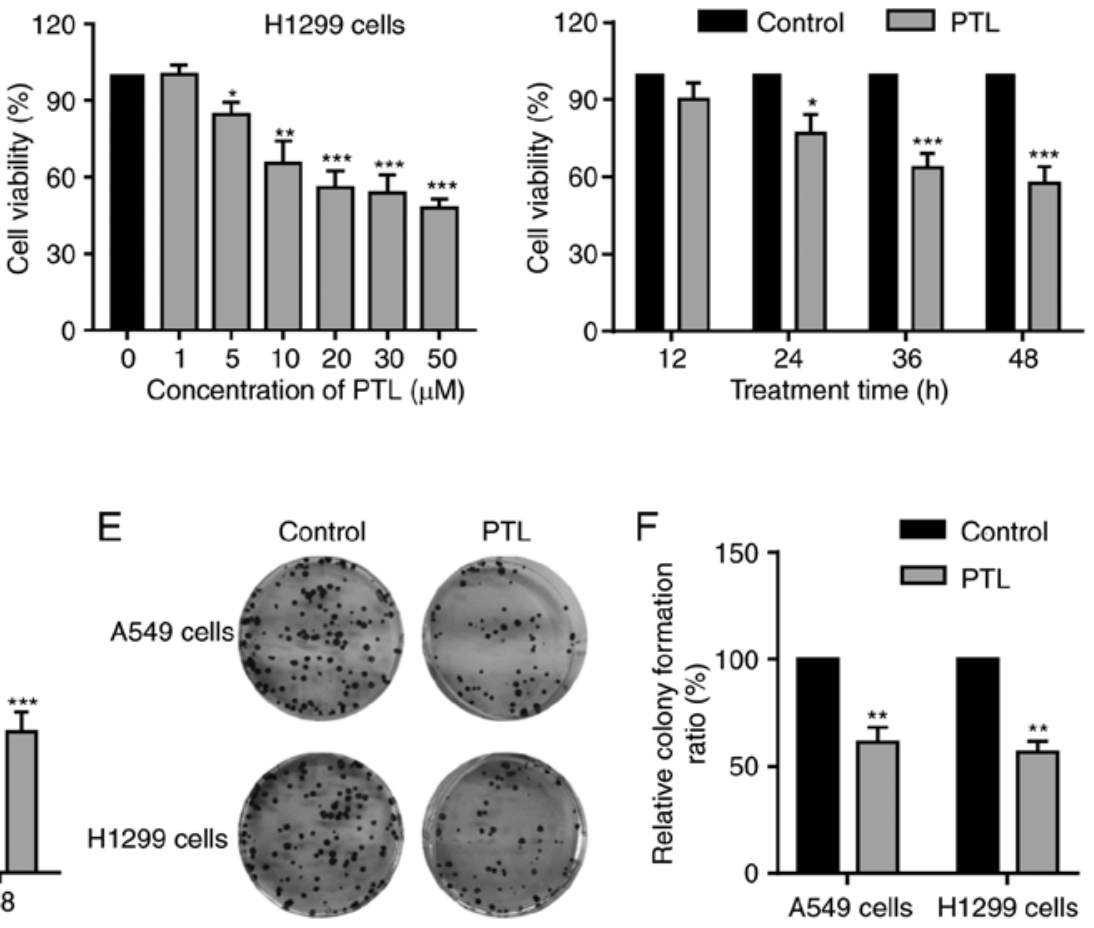

Figure 2. PTL treatment inhibits the proliferation of A549 and H1299 cells. (A) A549 and (B) H1299 cells were exposed to various concentrations (0-50 $\mu$ M) of PTL as indicated for $48 \mathrm{~h}$. (C) A549 and (D) H1299 cells were treated with $10 \mu \mathrm{M}$ PTL for 12, 24,36 and 48 h. A Cell Counting Kit-8 assay was performed to assess the cell viability. (E) Representative images of colonies formed by A549 and H1299 cells. Cells were treated with $10 \mu \mathrm{M}$ PTL for two weeks. (F) Summary plots of colony formation ratio in E. Values are expressed as the mean \pm standard error of the mean. ${ }^{*} \mathrm{P}<0.05,{ }^{* *} \mathrm{P}<0.01,{ }^{* * *} \mathrm{P}<0.001 \mathrm{vs}$. control group. PTL, parthenolide.

A

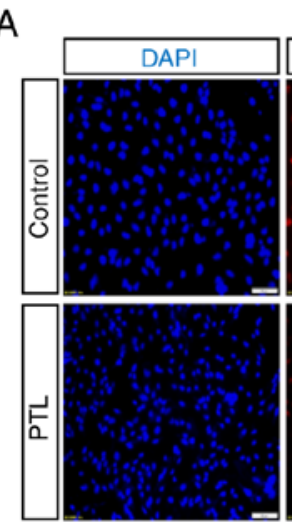

C

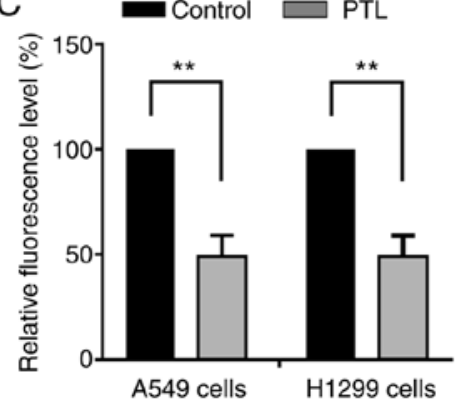

B

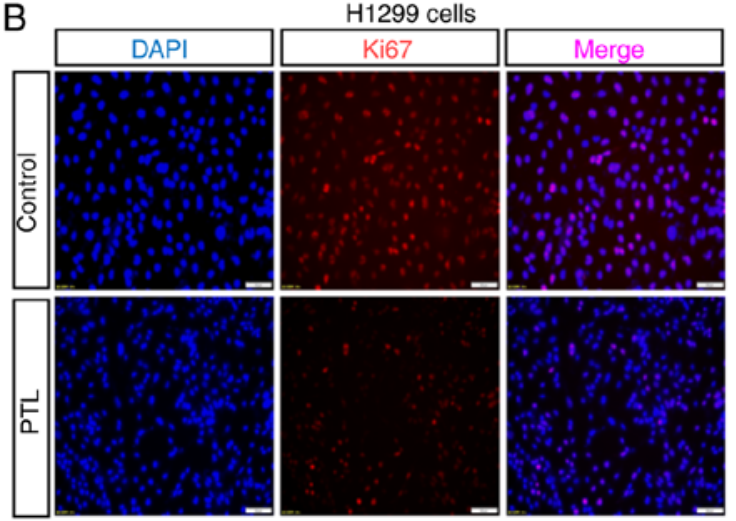

D
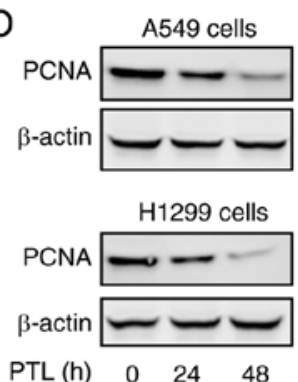

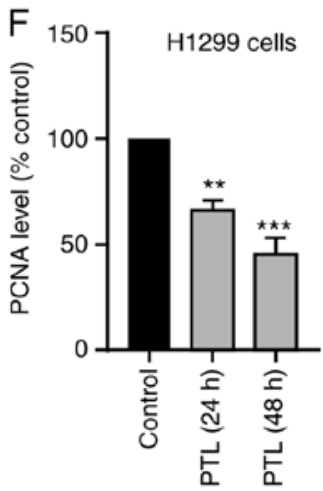

Figure 3. PTL inhibits Ki67 and PCNA expression in lung cancer cells. (A and B) Representative images of immunolabeling for Ki67 expression in (A) A549 and (B) H1299 cells (scale bar, $50 \mu \mathrm{m}$ ). (C) Statistical analysis of Ki67 expression from A and B. (D) Representative western blots for PCNA expression in A549 and H1299 cells after PTL treatment. (E and F) Quantitation of western blot results in (E) A549 and (F) H1299 cells. Values are expressed as the mean \pm standard error of the mean. ${ }^{* *} \mathrm{P}<0.01,{ }^{* * *} \mathrm{P}<0.001$ vs. control group. PTL, parthenolide; PCNA, proliferating cell nuclear antigen. 
A

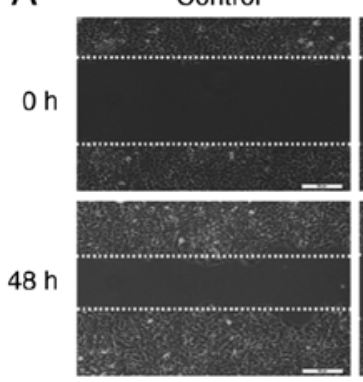

C

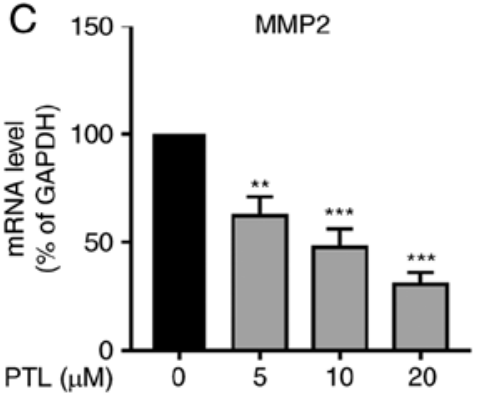

$5 \mu \mathrm{M}$

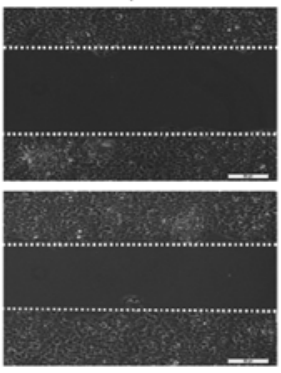

D 150

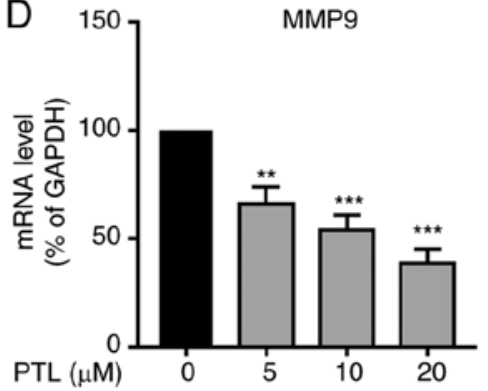

B Control

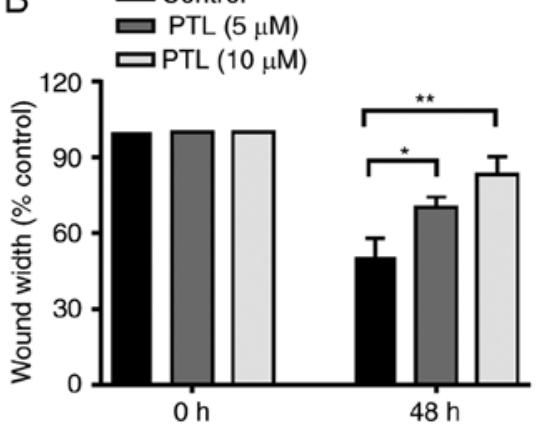

E

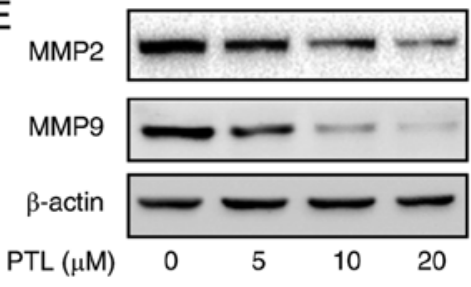

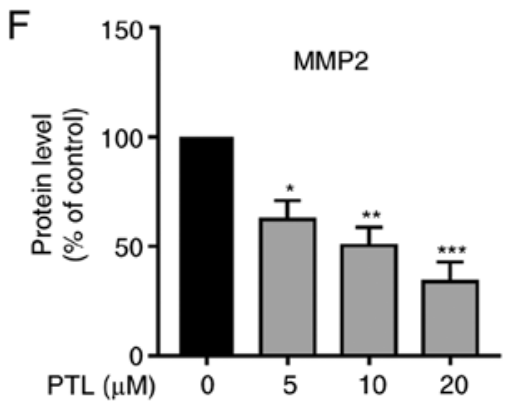

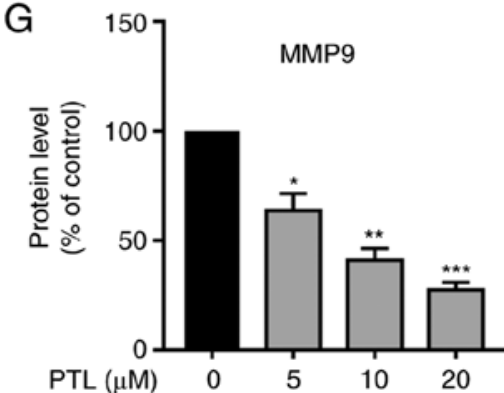

Figure 4. PTL markedly suppresses lung cancer cell migration. (A) Representative images displaying the effect of PTL on A549 cell migration (scale bar, $50 \mu \mathrm{m}$ ). (B) Relative wound width in the different groups of cells. (C and D) Cells were incubated with PTL for $48 \mathrm{~h}$, mRNA level of (C) MMP-2 and (D) MMP-9 were measured by reverse transcription-quantitative PCR. (E) Representative western blots of the protein levels of MMP-2 and MMP-9 in the cells following treatment with PTL. (F and G) Quantitative analysis of (F) MMP-2 and (G) MMP-9 protein expression. Values are expressed as the mean \pm standard error of the mean. ${ }^{*} \mathrm{P}<0.05,{ }^{* *} \mathrm{P}<0.01,{ }^{* * *} \mathrm{P}<0.001$ vs. control group. $\mathrm{PTL}$, parthenolide; MMP, matrix metalloproteinase.

metastasis, the effect of PTL on the migration of A549 cells was then examined by a using wound-healing assay. As presented in Fig. 4A, treatment with PTL at 5 and $10 \mu \mathrm{M}$ for 48 h significantly decreased the migration ability of the A549 cells compared with that in the control group (Fig. 4A and B).

Extracellular matrix (ECM) degradation by MMPs, such as MMP-2 and MMP-9, plays a pivotal role in the migration and metastasis of a variety of tumor types. It was therefore investigated whether PTL reduces the expression of MMP-2 and MMP-9 in lung cancer cells. Compared to the control group, PTL treatment for $48 \mathrm{~h}$ significantly downregulated the mRNA expression level of MMP-2 (Fig. 4C) and MMP-9 (Fig. 4D) in a dose-dependent manner. Furthermore, the protein levels of MMP-2 (Fig. 4E and F) and MMP-9 (Fig. 4E and G) were also significantly inhibited by PTL treatment.

PTL inhibits the activation of the IGF-1R/Akt/FoxO3 $\alpha$ pathway in A549 cells. The IGF-1R-mediated PI3K/Akt signaling cascade has a crucial role in the proliferation, survival, invasion and angiogenesis of multiple cancers (28). First, the effect of PTL on IGF-1R activation was explored. As presented in Fig. 5A, in comparison with the untreated cells, PTL induced a reduction in the phosphorylation level of IGF-1R (pIGF1-R), while it had no obvious effect on the total expression level of IGF-1R (Fig. 5B).

The effects of PTL on Akt phosphorylation were then assessed. Treatment of A549 cells with PTL significantly reduced Akt phosphorylation (pAkt) that showing a similar trend with IGF-1R (Fig. 5C). Furthermore, the effect of PTL on the expression of FoxO $3 \alpha$, a transcription factor and tumor suppressor, in A549 cells was determined. Of note, the phosphorylation of FoxO $3 \alpha$ (p FoxO3 $\alpha$ ) was also blocked by PTL (Fig. 5D). These results suggest that PTL significantly inhibited the IGF-1R/Akt/FoxO3 $\alpha$ signaling pathway in A549 cells.

PTL inhibits IGF-1-induced activation of the PI3K/Akt pathway in A549 cells. High circulating levels of IGF-1 are associated with a high risk in numerous types of cancer (19). It was first confirmed that IGF-1 markedly increased Akt and FoxO3 $\alpha$ phosphorylation (Fig. 6A-C). However, the increased phosphorylation levels of Akt (pAkt) and FoxO $3 \alpha$ (pFoxO $3 \alpha$ ) induced by IGF-1 were significantly attenuated by PTL pre-treatment (Fig. 6D-F). 

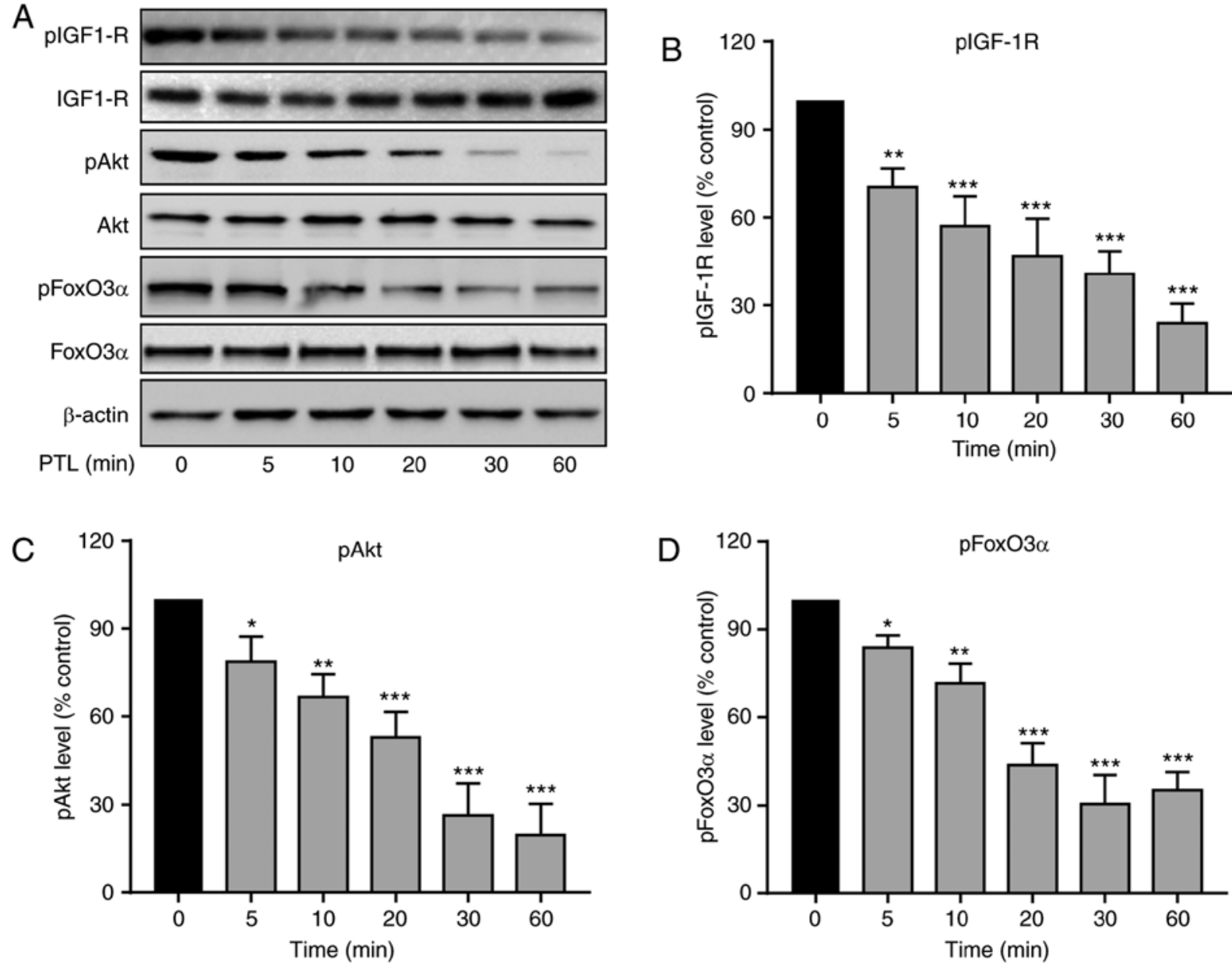

Figure 5. PTL inhibits IGF-1R, Akt and FoxO3 $\alpha$ phosphorylation in A549 cells. A549 cells were treated with PTL (10 $\mu \mathrm{M})$ for various durations (0-60 min) as indicated. (A) Representative western blots indicating IGF-1R, Akt and FoxO3 $\alpha$ phosphorylation induced by IGF-1. (B-D) Quantified phosphorylation levels of (B) IGF-1R, (C) Akt and (D) FoxO3 $\alpha$. Values are expressed as the mean \pm standard error of the mean. ${ }^{*} \mathrm{P}<0.05,{ }^{* *} \mathrm{P}<0.01,{ }^{* * *} \mathrm{P}<0.001$ vs. control group. IGF-1R, insulin-like growth factor 1 receptor; FoxO3 $\alpha$, forkhead box $\mathrm{O} 3 \alpha$; p, phosphorylated; PTL, parthenolide.

To verify that Akt and FoxO3 $\alpha$ phosphorylation was mediated by IGF-1R in A549 cells, A549 cells were pretreated with LY294002, an inhibitor of PI3K, for $30 \mathrm{~min}$, and the levels of p-Akt and p-FoxO3 $\alpha$ induced by IGF-1 were then detected. The results indicated that LY294002 markedly inhibited IGF-1-induced Akt and FoxO3 $\alpha$ phosphorylation (Fig. 6G-I), further confirming that Akt and FoxO3 $\alpha$ were downstream targets of PI3K in A549 cells.

PTL inhibits IGF-1-induced A549 cell proliferation and migration. The results revealing that PTL suppressed the cell proliferation and migration, as well as inhibited the phosphorylation of IGF-1R/PI3K/Akt/FoxO3 $\alpha$ in A549 cells, strongly suggest that the antitumor effect of PTL may be mediated by the IGF-1R/PI3K/Akt pathway. Thus, the effect of PTL on IGF-1-induced proliferation of A549 cells was then determined. As presented in Fig. 7A, IGF-1 significantly increased the proliferation of A549 cells, while pre-treatment with PTL significantly attenuated the IGF-1-induced cell proliferation (Fig. 7A). Similarly, PTL also significantly inhibited IGF-1-induced cell migration ability (Fig. 7B and C). Thus, the above results indicate that PTL inhibits the proliferation and migration of lung cancer cells by downregulating IGF-1R and its downstream signaling cascades.
PTL inhibits the growth of lung cancer in a mouse xenograft model. Considering the above in vitro results that PTL suppressed the proliferation and migration of lung cancer cells, the present study then set out to determine whether PTL is able to inhibit the growth of lung cancer in vivo. Nude mice were used to establish a lung cancer subcutaneous tumor xenograft model. Compared with the control group, PTL treatment resulted in a significant reduction in tumor growth and volume (Fig. 8A). During the entire treatment period, no apparent alteration in body weight was observed between the groups (Fig. 8B). Furthermore, after treatment, the tumors were dissected from each mouse to calculate the tumor weight in the different groups. The results suggested that the tumor weight in PTL-treated mice was significantly lower than that in the control group (Fig. 8C and D).

\section{Discussion}

In the present study, the anticancer effects of parthenolide (PTL), a natural product extracted from the plant Tanacetum parthenium (a flowering plant in the daisy family, Asteraceae) on the proliferation and migration of lung cancer cells, as well as the underlying molecular mechanisms, were investigated. The results indicated that PTL reduced the expression of 

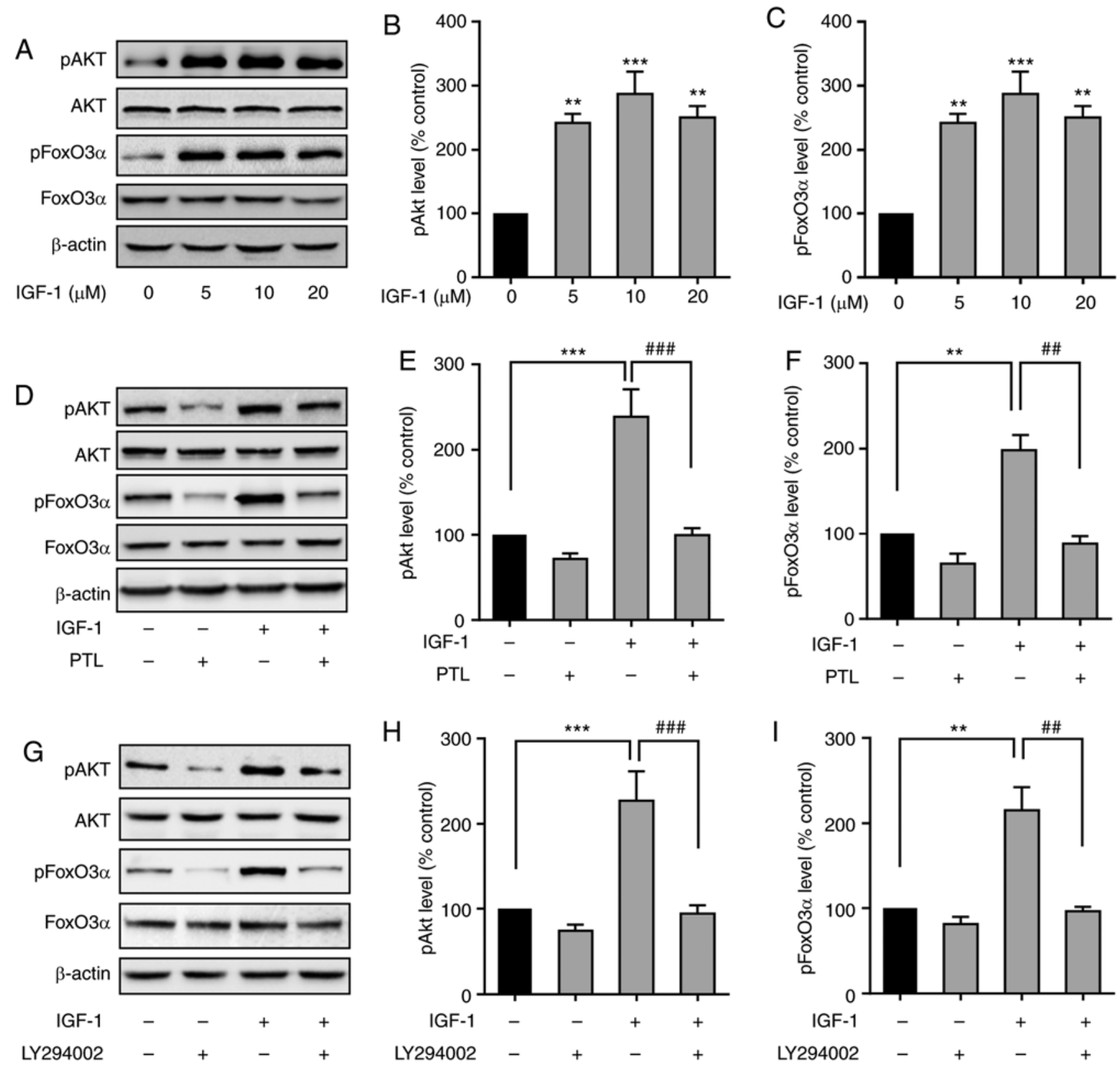

Figure 6. PTL blocks IGF-1-induced Akt and FoxO3 $\alpha$ phosphorylation in A549 cells. (A) Representative western blots indicating Akt and FoxO3 $\alpha$ phosphorylation induced by IGF-1. (B and C) Quantified phosphorylation levels of (B) Akt and (C) FoxO3 $\alpha$. (D) Representative western blots indicating the effects of PLT on IGF-1-induced Akt and FoxO3 $\alpha$ phosphorylation. (E and F) Quantified phosphorylation levels of (E) Akt and (F) FoxO3a. (G) Representative western blots indicating the effects of LY294002 on IGF-1-induced Akt and FoxO3 $\alpha$ phosphorylation. (H and I) Quantified phosphorylation levels of (H) Akt and (I) FoxO3 $\alpha$. Values are expressed as the mean \pm standard error of the mean. ${ }^{* *} \mathrm{P}<0.01,{ }^{* * *} \mathrm{P}<0.001$ vs. the control group; ${ }^{\# \#} \mathrm{P}<0.01,{ }^{\# \# \#} \mathrm{P}<0.001$ vs. the IGF-1-treated group. PTL, parthenolide; IGF-1R, insulin-like growth factor 1 receptor; FoxO3 $\alpha$, forkhead box O3 $\alpha$; p, phosphorylated.

matrix metalloproteinase (MMP)-2 and MMP-9, inhibited the IGF-1R/PI3K/Akt/FoxO3 $\alpha$ signaling pathway and consequently reduced the migration and proliferation of lung cancer cells.

Natural plant extracts provide a variety of drug candidates for cancer treatment. Accumulating evidence has indicated that PTL exerts anticancer effects mainly through interfering with the cancer progression process, including suppression of the proliferation and migration, or induction of cell apoptosis in various types of cancer cells $(29,30)$. The results of the present study indicated that PTL significantly suppressed the proliferation of A549 and H1299 lung cancer cells. Furthermore, molecular biology assays confirmed the anti-proliferation effect of PTL on those lung cancer cells. Of note, the expression of proliferating cell nuclear antigen (PCNA) protein was significantly decreased after PTL treatment. Since PCNA is found in the nucleus and has an essential role in numerous cellular processes, including DNA replication and cell-cycle progression (31), it has been accepted that PCNA expression may be used as a potential indicator for cancer prognosis (32). In addition, the expression of Ki67, a widely used marker of tumor aggressiveness and proliferation, was inhibited by PTL treatment. Furthermore, in the mouse xenograft model of leukemia induced by inoculating A549 cells into BALB/c mice, PTL significantly inhibited the tumor growth in vivo, strongly suggesting that PTL suppresses lung cancer growth. 

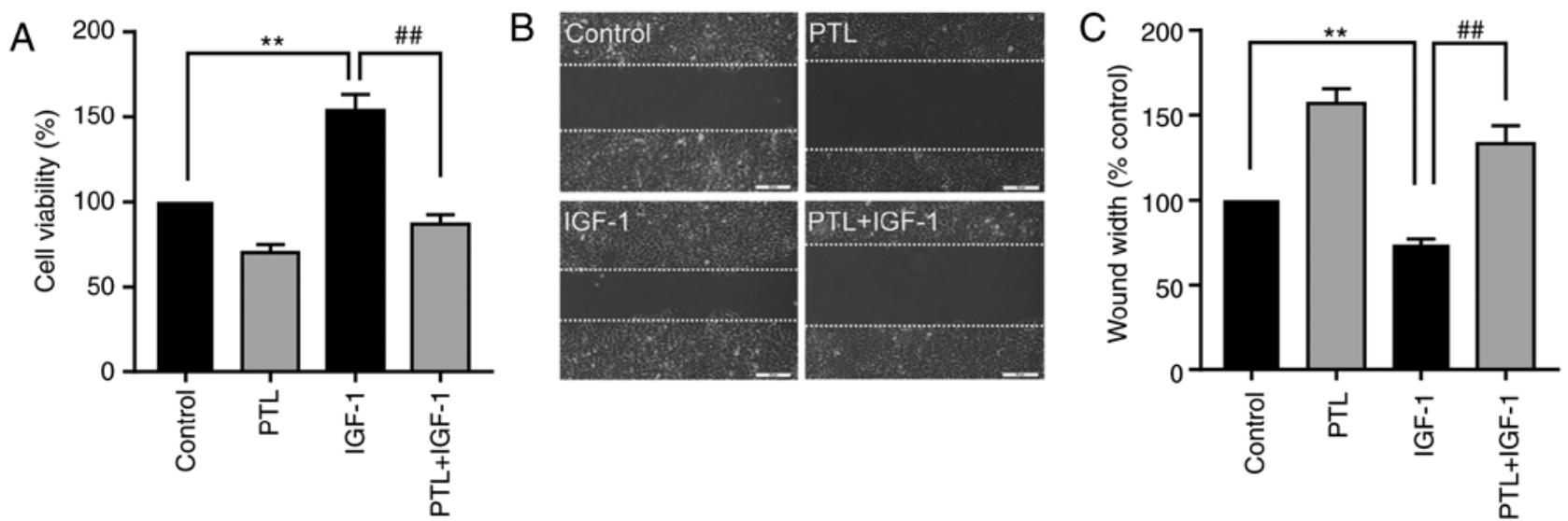

Figure 7. PTL inhibits IGF-1-induced A549 cell proliferation and migration. (A) Effect of PTL on the IGF-1-induced proliferation of A549 cells. (B) Representative images of the effect of PTL on IGF-1-induced migration of A549 cells (scale bar, $50 \mu \mathrm{m}$ ). (C) Relative wound width in the different groups of cells. Values are expressed as the mean \pm standard error of the mean. ${ }^{* *} \mathrm{P}<0.01$ vs. the control group; ${ }^{\# \#} \mathrm{P}<0.01$, vs. the IGF-1-treated group. PTL, parthenolide; IGF-1R, insulin-like growth factor 1 receptor.
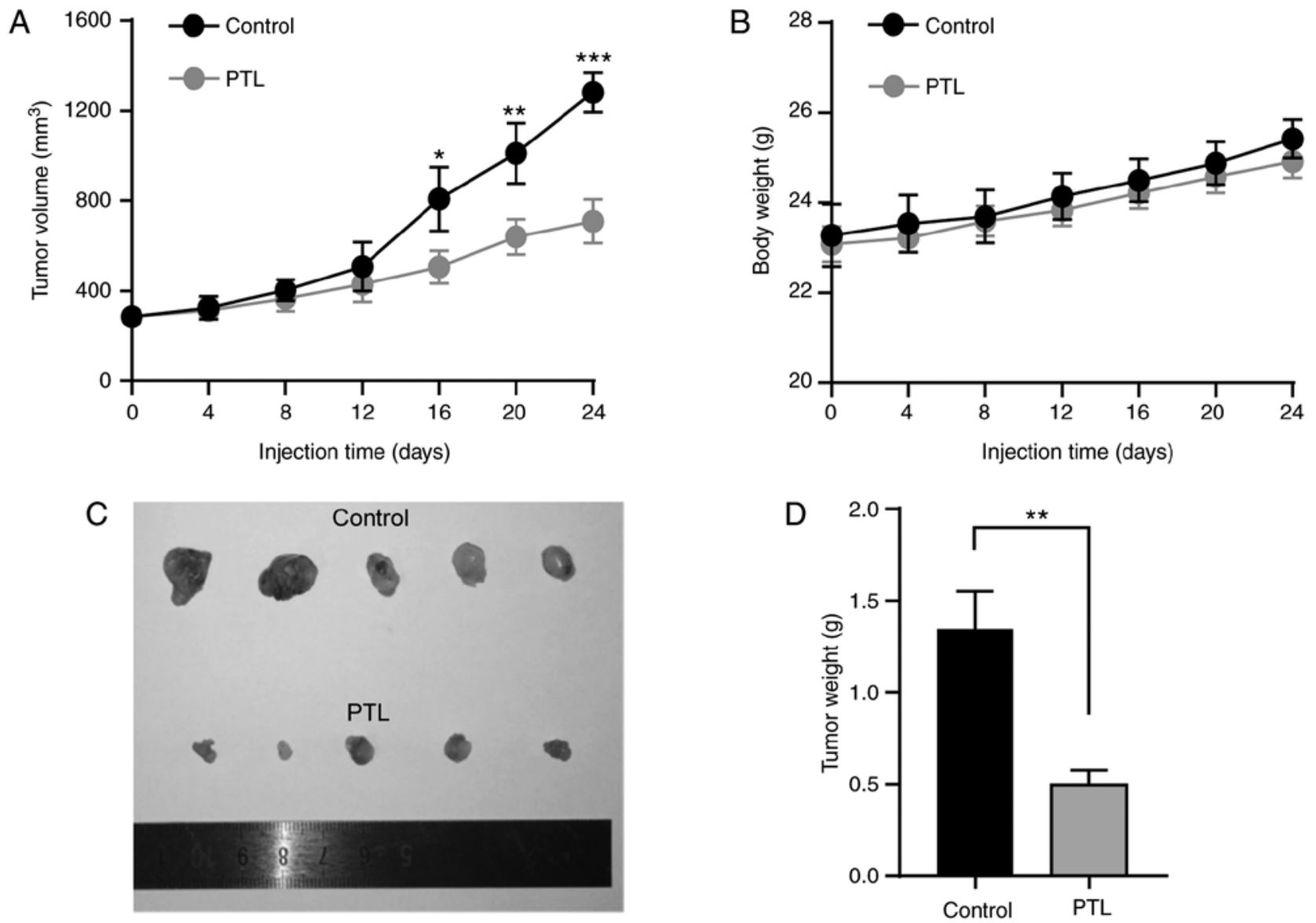

Figure 8. PTL suppresses tumor growth in a subcutaneous xenograft mouse model. (A) Curves of tumor growth in mice treated with PTL. (B) Body weight of mice subjected to PTL treatment up to day 24. (C) Representative images of tumors of mice obtained at the end of the experiment. (D) Mean weight of tumors in each group after PTL treatment $(\mathrm{n}=5)$. Values are expressed as the mean \pm standard error of the mean. ${ }^{*} \mathrm{P}<0.05,{ }^{, * *} \mathrm{P}<0.01,{ }^{* * * *} \mathrm{P}<0.001$ vs. the control group. PTL, parthenolide.

To demonstrate the mechanism by which PTL regulates lung cancer cell growth, the effects of PTL on insulin-like growth factor 1 (IGF-1) signaling, which is fundamental for growth, survival and migration, were evaluated. Increasing evidence indicates that the IGF-1 receptor (IGF-1R) is abnormally expressed in multiple cancer types (17), and it has thus been suggested as a potential anticancer target (33). Indeed, inhibitors targeting IGF-1 signaling have been assessed in clinical cancer trials, confirming IGF-1R as an effective therapeutic target (34). In addition, overexpression of IGF-1R has been determined in lung cancer and high IGF-1R expression is highly associated with an increased risk of developing 
malignant tumors, as well as with a low survival rate of patients with NSCLC after surgical resection (35). Therefore, targeting the IGF-1/IGF-1R has been recognized as an attractive strategy for the prevention and treatment of lung and other types of cancer. The present study indicated that PTL is effective in inhibiting IGF-1R phosphorylation. Furthermore, PTL treatment also blocked IGF-1-induced IGF-1R activation, as well as the proliferation of lung cancer cells. These results suggest that PTL may inhibit the growth of lung cancer cells by inhibiting IGF-1R signaling. However, it remains elusive how PTL acts to suppress IGF-1R phosphorylation, which requires to be explored in future research.

Accumulating evidence suggests that IGF-1R activation leads to PI3K/Akt activation, which is mostly involved in regulating the growth of cancer cells (29). In addition, clinical studies have revealed that PI3K/Akt activation is associated with tumor progression. Aberrant activation of the PI3K/Akt pathway confers drug resistance in a variety of cancer types (36). Of note, a previous study has demonstrated that PTL suppresses cell growth and induces apoptosis through attenuating the PI3K/Akt signaling cascade in cervical cancer cells (29). In the present study, it was confirmed that in lung cancer cells, PTL suppressed Akt phosphorylation and blocked IGF-1-induced Akt activation. In line with this, IGF-1-induced proliferation of A549 cells was also inhibited by PTL treatment, suggesting that PTL may be an attractive candidate for lung cancer therapy. In addition, PTL significantly inhibited the phosphorylation of transcription factor FoxO3 $\alpha$. However, the exact mechanisms of how PTL-induced de-phosphorylation of FoxO3 $\alpha$ functions to suppress lung cancer cell growth remain to be fully elucidated. Phosphorylation of FoxO3 $\alpha$ promotes its interaction with 14-3-3 protein, which ultimately leads to nuclear export to the cytoplasm to inhibit the transcriptional functions of FoxO3 $\alpha$ thus facilitating cell growth (37). By contrast, inhibition of the phosphorylation of PI3K/Akt induces de-phosphorylation of FoxO3 $\alpha$, leading to FoxO3 $\alpha$ nuclear translocation to arrest cell cycle progression and promote apoptosis. In future studies, it is required to demonstrate whether PTL-induced de-phosphorylation of FoxO3 $\alpha$ is responsible for enhancing apoptosis of lung cancer cells.

MMPs are calcium-dependent zinc-containing endopeptidases that have essential roles in degrading ECM and basement membranes. MMPs are widely known to be linked to the metastatic ability of cancer cells (38). Overexpression of MMP-2 and MMP-9 is frequently observed in tumor tissues and is highly associated with tumor metastasis and aggressiveness of various cancer types (39). Of note, the expression of MMP-2 and MMP-9 is upregulated, which contributes to the migration of A549 cancer cells during lung cancer progression (40). Consistent with this, the present study demonstrated that PTL markedly inhibited MMP-2 and MMP-9 expression, as well as attenuated IGF-1-induced migration of A549 cells. Of note, upregulation of MMP-2 and MMP-9 has been reported to be influenced by PI3K/Akt activation in numerous types of cancer cell, including human melanoma (41) and A549 cells (42), and this was consistent with the present result that PTL suppressed the PI3K/Akt pathway in A549 cells.

In summary, the present study demonstrated that PTL, a natural product isolated from the medicinal plant Tanacetum parthenium, not only inhibits the proliferation and migration of lung cancer cells in vitro, but also suppresses lung cancer growth in vivo. The anticancer effect of PTL may be exerted by blocking IGF-1R-mediated PI3K/Akt signaling cascades, including inhibition of FoxO3 $\alpha$ activity. Furthermore, PTL suppressed the migration of lung cancer cells, which involved changes in the expression of migration-associated genes, including MMP-2 and MMP-9. The present results suggest that PTL may be a potential candidate for lung cancer treatment.

\section{Acknowledgements}

Not applicable.

\section{Funding}

The present study was supported by grants from the National Natural Science Foundation of China (grant nos. 81960523, 81460444 and 81660391).

\section{Availability of data and materials}

The datasets used during the current study are available from the corresponding author upon reasonable request.

\section{Authors' contributions}

LS and WY conducted most of the experiments. GW, BY and FX analyzed the data. XG, JT and QZ performed the animal experiments. LZ and CC performed the immunofluorescence experiments. WZ conceived and designed the study. LS, WY and WZ wrote the manuscript. All authors read and approved the manuscript and agree to be accountable for all aspects of the research in ensuring that the accuracy or integrity of any part of the work are appropriately investigated and resolved.

\section{Ethics approval and consent to participate}

All animal experimentation was approved by the Ethics Committee of the First Affiliated Hospital of Nanchang University (Nanchang, Jiangxi, China).

\section{Patient consent for publication}

Not applicable.

\section{Competing interests}

The authors declare that they have no competing interests.

\section{References}

1. Bittoni MA, Focht BC, Clinton SK, Buckworth J and Harris RE: Prospective evaluation of $\mathrm{C}$-reactive protein, smoking and lung cancer death in the Third National Health and Nutrition Examination Survey. Int J Oncol 47: 1537-1544, 2015.

2. Nawaz K and Webster RM: The non-small-cell lung cancer drug market. Nat Rev Drug Discov 15: 229-230, 2016.

3. Di X, Jin X, Li R, Zhao M and Wang K: CircRNAs and lung cancer: Biomarkers and master regulators. Life Sci 220: 177-185, 2019.

4. Youlden DR, Cramb SM and Baade PD: The international epidemiology of lung cancer: Geographical distribution and secular trends. J Thorac Oncol 3: 819-831, 2008. 
5. Li LC and Kan LD: Traditional Chinese medicine for pulmonary fibrosis therapy: Progress and future prospects J Ethnopharmacol 198: 45-63, 2017.

6. Efferth T, Li PC, Konkimalla VS and Kaina B: From traditional Chinese medicine to rational cancer therapy. Trends Mol Med 13: 353-361, 2007.

7. Liu D, Chen L, Zhao H, Vaziri ND, Ma SC and Zhao YY: Small molecules from natural products targeting the $\mathrm{Wnt} / \beta$-catenin pathway as a therapeutic strategy. Biomed Pharmacother 117 108990, 2019.

8. Ghantous A, Sinjab A, Herceg Z and Darwiche N: Parthenolide: From plant shoots to cancer roots. Drug Discov Today 18 894-905, 2013.

9. Zhang S, Ong CN and Shen HM: Critical roles of intracellular thiols and calcium in parthenolide-induced apoptosis in human colorectal cancer cells. Cancer Lett 208: 143-153, 2004.

10. Liu YC, Kim SL, Park YR, Lee ST and Kim SW: Parthenolide promotes apoptotic cell death and inhibits the migration and invasion of SW620 cells. Intest Res 15: 174-181, 2017.

11. Liu JW, Cai MX, Xin Y, Wu QS, Ma J, Yang P, Xie HY and Huang DS: Parthenolide induces proliferation inhibition and apoptosis of pancreatic cancer cells in vitro. J Exp Clin Cancer Res 29: 108, 2010.

12. Wyrębska A, Szymański J, Gach K, Piekielna J, Koszuk J, Janecki T and Janecka A: Apoptosis-mediated cytotoxic effects of parthenolide and the new synthetic analog MZ-6 on two breast cancer cell lines. Mol Biol Rep 40: 1655-1663, 2013.

13. Jafari N, Nazeri S and Enferadi ST: Parthenolide reduces metastasis by inhibition of vimentin expression and induces apoptosis by suppression elongation factor $\alpha-1$ expression Phytomedicine 41: 67-73, 2018

14. Talib WH and Al Kury LT: Parthenolide inhibits tumorpromoting effects of nicotine in lung cancer by inducing P53-dependent apoptosis and inhibiting VEGF expression. Biomed Pharmacother 107: 1488-1495, 2018.

15. Lin M, Bi H, Yan Y, Huang W, Zhang G, Zhang G, Tang S, Liu Y Zhang L, Ma J and Zhang J: Parthenolide suppresses non-smal cell lung cancer GLC-82 cells growth via B-Raf/MAPK/Erk pathway. Oncotarget 8: 23436-23447, 2017

16. Regad T: Targeting RTK signaling pathways in cancer. Cancers (Basel) 7: 1758-1784, 2015.

17. Denduluri SK, Idowu O, Wang Z, Liao Z, Yan Z, Mohammed MK Ye J, Wei Q, Wang J, Zhao L and Luu HH: Insulin-like growth factor (IGF) signaling in tumorigenesis and the development of cancer drug resistance. Genes Dis 2: 13-25, 2015.

18. Casa AJ, Dearth RK, Litzenburger BC, Lee AV and Cui X: The type I insulin-like growth factor receptor pathway: A key player in cancer therapeutic resistance. Front Biosci 13: 3273-3287, 2008 .

19. Weroha SJ and Haluska P: The insulin-like growth factor system in cancer. Endocrinol Metab Clin North Am 41: 335-350, vi, 2012.

20. Mayer IA and Arteaga CL: The PI3K/AKT pathway as a target for cancer Treatment. Annu Rev Med 67: 11-28, 2016.

21. Farhan M, Wang H, Gaur U, Little PJ, Xu J and Zheng W: FOXO signaling pathways as therapeutic targets in cancer. Int J Biol Sci 13: 815-827, 2017.

22. Coomans de Brachène $\mathrm{A}$ and Demoulin JB: FOXO transcription factors in cancer development and therapy. Cell Mol Life Sci 73: $1159-1172,2016$

23. Hornsveld M, Dansen TB, Derksen PW and Burgering BMT: Re-evaluating the role of FOXOs in cancer. Semin Cancer Biol 50: 90-100, 2018

24. Zhang J, Wen G, Sun L, Yuan W, Wang R, Zeng Q, Zhang G and Yu B: Cryptotanshinone inhibits cellular proliferation of human lung cancer cells through downregulation of IGF-1R/PI3K/Akt signaling pathway. Oncol Rep 40: 2926-2934, 2018.
25. Livak KJ and Schmittgen TD: Analysis of relative gene expression data using real-time quantitative PCR and the 2(-Delta Delta C(T)) method. Methods 25: 402-408, 2001.

26. Nakabayashi $\mathrm{H}$ and Shimizu K: Involvement of Akt/NF- $\kappa \mathrm{B}$ pathway in antitumor effects of parthenolide on glioblastoma cells in vitro and in vivo. BMC Cancer 12: 453, 2012.

27. Jakobsen JN and Sørensen JB: Clinical impact of ki-67 labeling index in non-small cell lung cancer. Lung Cancer 79: 1-7, 2013.

28. Altorki NK, Markowitz GJ, Gao D, Port JL, Saxena A, Stiles B, McGraw T and Mittal V: The lung microenvironment: An important regulator of tumour growth and metastasis. Nat Rev Cancer 19: 9-31, 2019.

29. Jeyamohan S, Moorthy RK, Kannan MK and Arockiam AJV: Parthenolide induces apoptosis and autophagy through the suppression of PI3K/Akt signaling pathway in cervical cancer. Biotechnol Lett 38: 1251-1260, 2016.

30. Yu HJ, Jung JY, Jeong JH, Cho SD and Lee JS: Induction of apoptosis by parthenolide in human oral cancer cell lines and tumor xenografts. Oral Oncol 51: 602-609, 2015.

31. Stoimenov I and Helleday T: PCNA on the crossroad of cancer. Biochem Soc Trans 37 7(Pt 3): 605-613, 2009.

32. Juríková M, Danihel L, Polák S and Varga I: Ki67, PCNA, and MCM proteins: Markers of proliferation in the diagnosis of breast cancer. Acta Histochem 118: 544-552, 2016.

33. Peled N, Wynes MW, Ikeda N, Ohira T, Yoshida K, Qian J, Ilouze M, Brenner R, Kato Y, Mascaux C and Hirsch FR: Insulin-like growth factor-1 receptor (IGF-1R) as a biomarker for resistance to the tyrosine kinase inhibitor gefitinib in non-small cell lung cancer. Cell Oncol (Dordr) 36: 277-288, 2013.

34. Singh P, Alex JM and Bast F: Insulin receptor (IR) and insulin-like growth factor receptor 1 (IGF-1R) signaling systems: Novel treatment strategies for cancer. Med Oncol 31: 805, 2014.

35. Gong Y, Yao E, Shen R, Goel A, Arcila M, Teruya-Feldstein J, Zakowski MF, Frankel S, Peifer M, Thomas RK, et al: High expression levels of total IGF-1R and sensitivity of NSCLC cells in vitro to an anti-IGF-1R antibody (R1507). PLoS One 4: e7273, 2009.

36. Yang J, Nie J, Ma X, Wei Y, Peng Y and Wei X: Targeting PI3K in cancer: Mechanisms and advances in clinical trials. Mol Cancer 18: 26, 2019.

37. Tzivion G, Dobson M and Ramakrishnan G: FoxO transcription factors; Regulation by AKT and 14-3-3 proteins. Biochim Biophys Acta 1813: 1938-1945, 2011.

38. Piperigkou Z, Manou D, Karamanou K and Theocharis AD: Strategies to target matrix metalloproteinases as therapeutic approach in cancer. Methods Mol Biol 1731: 325-348, 2018.

39. Cathcart J, Pulkoski-Gross A and Cao J: Targeting matrix metalloproteinases in cancer: Bringing new life to old ideas. Genes Dis 2: 26-34, 2015.

40. Hu P, He J, Liu S, Wang M, Pan B and Zhang W: $\beta 2$-adrenergic receptor activation promotes the proliferation of A549 lung cancer cells via the ERK1/2/CREB pathway. Oncol Rep 36: $1757-1763,2016$

41. Yao X, Jiang W, Yu D and Yan Z: Luteolin inhibits proliferation and induces apoptosis of human melanoma cells in vivo and in vitro by suppressing MMP-2 and MMP-9 through the PI3K/AKT pathway. Food Funct 10: 703-712, 2019.

42. Gao Y, Guan Z, Chen J, Xie H, Yang Z, Fan J, Wang X and Li L: CXCL5/CXCR2 axis promotes bladder cancer cell migration and invasion by activating PI3K/AKT-induced upregulation of MMP2/MMP9. Int J Oncol 47: 690-700, 2015. 